\title{
Implementation of A Psychiatric Consultation for Healthcare Workers During First Wave of COVID-19 Outbreak: A Qualitative and Quantitative Analysis.
}

\section{Lamyae BENZAKOUR ( $\square$ lamyae.benzakour@hcuge.ch )}

https://orcid.org/0000-0002-1298-9659

\section{Gérard Langlois}

Geneva University Hospitals: Hopitaux Universitaires Geneve

\section{Verena Marini}

Geneva University Hospitals: Hopitaux Universitaires Geneve

\section{Alexandra Groz}

Geneva University Hospitals: Hopitaux Universitaires Geneve

\section{Chiara Chiabotto}

Geneva University Hospitals: Hopitaux Universitaires Geneve

\section{Diana Apetrei}

Geneva University Hospitals: Hopitaux Universitaires Geneve

\section{Bruno Corneau}

Geneva University Hospitals: Hopitaux Universitaires Geneve

\section{Guido Bondolfi}

Geneva University Hospitals: Hopitaux Universitaires Geneve

\section{Research article}

Keywords: healthcare workers, COVID-19, psychiatric consultation, burnout, PTSD, stress.

Posted Date: August 31st, 2021

DOI: https://doi.org/10.21203/rs.3.rs-856222/v1

License: (c) (1) This work is licensed under a Creative Commons Attribution 4.0 International License. Read Full License 


\section{Abstract}

Background:Prevention and management strategies of mental suffering in healthcare workers appeared as important challenges during COVID-19 pandemic. This article aims to 1) Identify potential psychiatric disorders for healthcare workers during the first wave of the COVID-19 outbreak; 2) Describe the implementation of a psychiatric consultation for healthcare workers; 3 ) Present the activity report of this consultation; 4) Analyze and learn from this experience.

Methods: The authors identified specific risks for healthcare workers mental health to be anxiety, depression, burnout and traumatic symptoms. The authors performed a retrospective quantitative analysis of socio-demographic and clinical data, in addition to psychiatric scales scores for the main potential psychiatric risks (PDI, PDEQ, PCL-5, HADS, MBI-HSS) and post-hoc qualitative analysis of written interviews. Means and frequencies were calculated for all the variables collected.

Results: 25 healthcare workers were consulted between 19 th March 2020 and 12 th June 2020, 18 who accepted to participate to the study. Authors found $78.57 \%$ presented high peritraumatic dissociation, $78.57 \%$, high peritraumatic distress, $68.75 \%$, severe anxiety symptoms, and $31.25 \%$, severe depression symptoms. Concerning burnout, authors found $35.29 \%$ had a moderate,and $23.53 \%$ a high level of Emotional Exhaustion; 17.65\%, a moderate, and 23.53\%, a high level of Depersonalization; $11.76 \%$, a low, and $35.29 \%$, a moderate level of Personal Achievement. In the qualitative analysis of the written interview, authors found a direct link with COVID-19, primarily concerning traumatic stressors, and secondarily with work-related stress.

Conclusions: This study confirms the psychiatric consequences during the first wave of the COVID-19 epidemic for healthcare workers. These results suggest that for healthcare workers, early detection of traumatic reactions, valorization of individual effort, and limitations on work overload, are potential key preventative measures.

\section{Background}

The outbreak of COronaVIrus Disease-19 (COVID-19) emerged in December 2019 in Wuhan (China) and has so far consisted of three waves havealready given rise in the world to $162,500,000$ confirmed cases, and has killed 3,369,259 as of May 2021 [1].To date, in Switzerland, 674,138 cases have been laboratoryconfirmed (7,833/100,000 inhabitants) and 10,080 deaths (117.13/100,000 inhabitants) caused by COVID-19, have been recorded [2]. From the pandemic's onset, healthcare workershave needed to adaptto this unprecedented situation to avoid hospital saturation, and limit both deaths and severe complications. For HCW, uncertainty about the length of the pandemic, the need to adapt to new care management due to the outbreak, and the lack of knowledge about COVID-19, were the most prominent stress factors. These challenges were identified early-on as risk factors of psychological suffering for hospital workers[3]. During the initial lockdown period from March 16to April 19 2020, HCW were confronted by the virus in the name of the collective good and quicklybecame, by force of circumstance, 
the "soldierson the frontline". In many countries, this image of HCWwas solidified by media and societal rituals, manifested by applause given at the end of each day. Within this context, it may have been difficult for HCW to recognize and acknowledge their psychological suffering.

The first published studies on psychological suffering of HCWduring the COVID-19 pandemic confirmed the existence of this heavy psychological burden, especially for anxiety, depression, and insomnia [4] [5].The influence on mental symptoms of age, gender, occupation, specialization, the types of activity performed, and their proximity to COVID-19 patients were also highlighted [4][5].Moreover, Post-Traumatic Stress Disorder (PTSD) risk in HCWduring previous coronavirus epidemicswas highlighted, as well as duringthe COVID-19 pandemic[6].

Certain variables were found to be particularly relevant as PTSD risk factors,such as the female sex, older age, exposure level, working role, years of work experience, social and work support, job organization, presence of quarantine, marital status, and resilience factors, such as coping styles and social support [6].

At the University hospital in Geneva, in the beginning of the first wave of COVID-19,we implemented several strategies in order to prevent and manage early psychological suffering among HCW. Our paper aims to 1) Show how potential psychiatric disorders for healthcare workers during the first wave of the COVID-19 outbreak were identified; 2) Describe the implementation of a psychiatric consultation service for healthcare workers; 3) Present an activity report of this consultation; 4) Analyze and learn from this experience for the future.

\section{Methods}

a. Identification of specific risks for mental health for healthcare workers in the beginning of the first wave of the COVID-19 pandemic

Prior to implementing a consultation service(CovidPsy) for HCW, we attempted to identify potential mental health risks to design our consultation methodology and adapt the terms of care that could be offered. We identified these using data from previous respiratory pandemicsof Severe Acute Respiratory Syndrome (SARS) in 2002-2003 and Middle East Respiratory Syndrome (MERS) in 2012,information from foreign media outlets, such as those in China where the pandemic began, adapting it to our own needshaving only limited experience with the virus in our own university hospital before implementing the CovidPsy consultation. We distinguished two main categories of stress factors: a) work-related stress during COVID-19 outbreak; b) direct stress consequences of COVID-19.

i. Work-related stress during the COVID-19 outbreak

Risk of loss of control

Faced with an uncontrollableviral outbreak and its treatment, HCW might have felt powerless to help their patients. For example, as the influx of patients increased, urgent improvised decisions had to be made to 
spare care resources, which at the time were almost entirely dedicated to managing COVID-19, and specifically implementing algorithms to prioritize care. As a result, the clinical activity of HCW was brutally and rapidly transformed, shaking the very basis of their professional identity. For many, time stood still from the start of this first wave of the health crisis. Unpredictability contaminated all aspects of the HCW's daily life, and especially when exposed to certain COVID-19 patients who experienced rapid deterioration.

\section{Risk of work intensity}

In our university hospital, HCW were "requisitioned", meaning that their vacation was suspended for an indefinite period of time, and were given in rare instances, time to decompress. Faced with difficulties anticipating healthcare resource needs during this unprecedented crisis, as well as absenteeism linked to staff contamination by SARS-CoV-2, HCW had to change medical units accordingly, regularly modifying their schedule, thus creating very unstable and intense working conditions.

\section{Risk of loss of sense}

In light of the pandemic, non-COVID clinical activities had to be abruptly suspended, giving the impression of patient abandonment. The question of patient triage arose, on the grounds of efficiency: COVID patients who needed to be given priority in intensive care units were those for whom an improvement in prognosis was considered more likely. Therefore, this management priority for COVID-19, to the detriment of other healthcare activities and their consequences [7]. In some instances, these situationsled to value conflictsand a profound loss of sense in many HCW who risked to no longer feel useful in the exercise of their profession.

\section{Risk of burnout}

Finally, during the first wave of the COVID-19 outbreak, concomitant losses of control and sense, as well as workload, increased, which are recognized as important factors of burnout as described by Maslach [8]. HCW are known to be exposed at higher risk of burnout and their complications [9] and some work factors such as lack of input or control for physicians, excessive workloads, inefficient work processes, clerical burdens have been identified for physicians [10].For these reasons, we considered it was important to investigate burnout and treat it in the context of this psychiatric consultation for HCW.

\section{ii. Direct stress consequences of COVID-19}

\section{An unpredictable virus}

In the course of their work, HCW were exposed to potential contamination by SARS-CoV-2 and subsequent increased risk of transmission to loved ones. The contradictory information surrounding the subject contributed to the caregivers' insecurity, as it was objectively impossible to guarantee protection, due to a lack of knowledge on the transmission modes of the virus. 
Specifically, HCW could find themselves faced with unpredictable, sudden, and numerous deaths, quantities they were not accustomed to, including those in intensive care units,even if they had previous experience with critical situations. In their personal lives, like the rest of the general population, many HCW were alsoimpacted due to the illness and deaths of those closest to them.

\section{A risk of social isolation outside of work}

The confluence for manyHCW of significant stress, the prioritization of professional duties above all others,and the restriction of social contacts and leisure time outside the hospital,has contributed to increasesin the risk for psychological distress such as anxiety, depression and traumatic experience during this period. Furthermore, holidays for hospital staff were abolished in most countries. In some cases, HCW were beginning to report situations of very painful rejection from their relatives for fear of contamination.

\section{A risk of stress related disorders and psychotraumatic sequelae}

Faced with a highly stressful situation, the subject's coping skills may be overwhelmed and give rise to reactive depressive or anxious symptoms. Depending on the level of personal resources available to mobilize coping strategies, and confronted with the same situation, some workers will develop anxious and / or depressive symptoms, whereas others may be able to adapt. Interindividual variability in reactions to a very stressful and unprecedented situation such as the COVID-19 pandemic was expected.

This COVID-19 context created situations of potential or actual death of patients,which meet the definition of traumatic events, as defined in the Diagnostic and Statistical Manual of Mental Disorders (DSM-5) [11].Subjects who had a history of trauma, either related or not to their professional activity, and/or a psychiatric history of depression, had a greater vulnerability for the risk of acute stress disorder (ASD) or post-traumatic stress disorder(PTSD) [12].Importantly, PTSD risk factors have been well studied during previous Coronavirus epidemics [13]. On the basis of past experiences and the identification of risk factors directly due to the COVID-19, a risk of PTSD in HCW seemed to be important and warranted further investigation. PTSD is associated with a poor prognosis and an important risk of comorbidities such as substance and alcohol abuse[14]. Prevention strategies are known to reduce the risk of chronic evolution to PTSD, such as an early identification and care for people at risk for PTSD even if the effectiveness of some interventions like cognitive-behavioral therapies (CBT), Eyes Movement Desensitization Reprocessing (EMDR), and pharmacological strategies,requires further study[15] [16].

\section{An opportunity of resilience and post-traumatic growth}

Important emphasis is placed on the potential psychiatric complications of this health crisis. However, in traumatic situations, psychological benefits grouped under the emerging notion of post-traumatic growth, can on the contrary develop in the aftermath of a traumatic event. Authors of a qualitative review on disaster-exposed organizations identified several protective factors after a disaster: training, experience, and perceived (personal) competence; social support; and effective coping strategies. Post-traumatic 
growth can provide a greater appreciation of life andrelationships, enhancing self-esteem and providing a sense of accomplishment and better understanding of an individual's work[17]. The exploration of these protective factors appeared as important of the risk factors for PTSD in time of COVID-19.

\section{b. Implementation of the CovidPsy psychiatric consultation}

\section{i. Establishment of the permanence CovidPsy}

Recommendations for setting up support systems for caregivers were quickly disseminated by World Health Organization at the start of the crisis [3][18]based on previous epidemics, highlighting the need to organize system for the prevention and the management of the mental suffering of the HCW.Within a few days in our hospital, the CovidPsy psychiatric consultation service was not only established, but a hotline, support psychologist positions in the COVID units, and hypnosis sessions were deployed. Material aids were offered such as parking spaces, accommodation in hotels for people who lived far away, and free meals.

The service of liaison psychiatry, the staff health service and Health Care Directorate, receivedan official mandate the 16h of March, 2020 from the medical director of university Hospital of Geneva and three days later, the $19^{\text {th }}$ of March, a psychiatricconsultation was offered to the hospital workers. The liaison psychiatry team usually works with hospitalized or outpatient patients for somatic pathologies and is not supposed to take care of the mental health of employees. However, in the psychiatry liaison chart, we are very aware of the importance of being attentive to the psychiatric impact of some clinical situations on their mental health and its potential impact on their work, and we have found that this demand made sense for us in the heart of this crisis. After the first step, when we received an official mandate, we made a request to two consultation offices. We implemented a 7 day a week, 9 AM to 6 PM consultation service, to receive any HCWrequesting help,free of charge and without an appointment. Furthermore, HCW were able toreach the hotline for an initialscreening and could then be referred directly to the consultation office. We chose a name and conceived of a framework for the consultation. We werereinforced by medical staff from our other psychiatric services within the department, in addition toprivate psychiatrists, and benefited from our close collaboration with clinical nursing specialists. A common network spacewas set up to exchange informationand share our calendars, as a way of referral for each consultation. Wecontacted the hospital communication service to widely disseminatethe information on the modalities of this service to hospital collaborators,regarding the opening of psychiatric consultation. Once per week, acoordination meeting was helf with the people in charge of the support systems (psychiatric consultation, hotline, psychologists support). Moreover, several training sessions were provided to the team, recalling important theoretical-clinical basis for colleagues who were less familiar with this clinical field and to transmit guidelines for the permanence, from the reception of the healthcare worker to the end of the care. We additionally provided sessions on advice for preventing exhaustion and vicarious trauma for the psychiatric consultation team who were exposed to heavy emotional burden arising fromcaring for their colleagues as well as to the global effect of the pandemic. Daily intervision sessions were organized to analyze and discuss the clinical situations and their care. 


\section{ii. Interventions}

The psychiatric consultation team was composed of hospital and private psychiatrists- psychotherapists, and clinical specialist nursesin psychiatry,whose usual clinical activity was reduced, thanks to the solidarity of other psychiatric services and the Health Care Directorate.The final team for the psychiatric consultation was composed of 9 psychiatrists- psychotherapists and 8 clinical specialist nurses in psychiatry to ensure the presence of at least one psychiatrist-psychotherapist and nurse at all times during opening hours. The psychiatric intervention policies (guidelines for the intervention, organization of the permanence, and establishment of the duty schedule) were defined. Our crisis intervention and algorithm modelswere inspired by disaster psychiatry [19]. They consisted of a preventive model based on the identification of traumatic stressors and high-risk subjects of psychological suffering[12].

Based on data and the knowledge from previous epidemics of mental health is impacted in hospital workers, we identified the following risks: 1) Burnout; 2) Trauma disorders (acute stress disorder trauma, vicarious trauma, post-trauma stress disorder); 4) Anxiety and depression symptoms. We proposed a systematic screening of these risks at the beginning of the consultation using the French version of The Pocket Guide to the DSM-5 TMDiagnostic Exam whose licence has been obtained for each survey [20] and completed the evaluation with some questions on psychiatric history, their family and social circles, and working conditions. Depending on this evaluation, this was followed by a personalized therapeutic interventionusing specific guidelines.Consultations were carried out by a psychiatrist-psychotherapists and a clinical specialist nurse in psychiatry to encourage complementary interventions and to partition emotional burden in a face-to-face. If acute stress symptoms were identified, recommended interventions after a traumatic event such as defusing intervention, psychoeducation intervention on PTSD and/orEyes Movement Desensitization Reprocessing (EMDR) for recent trauma were provided. If burnout symptoms were identified, we gave feedback about these symptoms to the hospital workers directly and suggested asick leave. In front of anxiety symptoms and/or acute stress symptoms, we used stress management tools like safe place, cardiac coherence and basic mindfulness. For all the clinical situations, analyses of stress factors were conducted with the person who consulted and a search of strategies to cope with them was undertaken. Personal resources were sought and reinforced as much as possible. Medications could be also be prescribed depending on the psychiatric evaluation. We proposed short interventions which should not exceed three consultations with a few exceptions. We organized a referral for another follow-up if required because the HCW was not clinically sufficiently improved (private psychiatristpsychotherapistor psychologist-psychotherapistor consultation center depending on the hospital).

\section{c. Systematic data collection}

We collected personal data (data birth, phone number, marital status, phone number, mail), information on working conditions (position held, department, work in COVID yard, change of service due to COVID-19 outbreak, ...), medical and psychiatric history and previous trauma, risk factors for severe forms of COVID19 , contamination by SARS-CoV-2. 


\section{d. Measures}

We chose to use a systematic screening with scales to look for these risks before each consultation to adapt the intervention to the needs of the worker. HCWcompleted different validated tools in their French versions to look for main psychiatric situations that were expected, before the intervention (methods previously described): 1) Maslach Burnout Inventory-Human Services Survey (MBI-HSS) consisted in three dimensions: Emotional Exhaustion (EE), Depersonalization (DP) and Personal Achievement (PA). The cutoff points of the MBI-HSS were $\leq 21$ and $\leq 32$ for the EE subscale, $\leq 23$ and $\leq 30$ for the reduced (PA) subscale, $\leq 6$ and $\leq 12$ for the (DP) subscale, and $\leq 15$ and $\leq 17$ (i.e., low, moderate, and high level) [21][22]. The level of burnout was considered high if EE was $\geq 27$, PA was $\leq 21$, and DP was $\geq 13$; moderate if EE was 17-26, PA was 38-22, and DP was 7-12; and low if EE was $\leq 16$, PA was $\geq 39$, and DP was $\leq 6$; 2) Hospital Anxiety and Depression Scale (HADS) which assesses transdiagnostic symptoms of anxiety and depression in patients with a somatic disorder, using a cutoff total score of 11 for anxiety and for depression[23]. 3) Peritraumatic distress inventory (PDI)which screens for distress symptoms during and immediately following a traumatic event,using a cutoff at 15 to identify a high risk of future PTSD[24] [25], 4)Peritraumatic Dissociative Experiences Questionnaire (PDEQ) which screens for dissociative symptoms such as depersonalization and derealization during and immediately following a traumatic event, using a cutoff at 15 to identify a high risk of future PTSD[26] [27]; 5) Posttraumatic Stress disorder Checklist for DSM-5 (PCL-5) which assesses current symptoms of PTSD, using a cutoff score of 33 to identify a PTSD diagnosis which was giveninstead of PDI and PDEQ only in the case of PTSD diagnosis made in the presence of PTSD criteria present more than one month, , to assessPTSD severity[28] [29].

\section{e. Participants}

All HCW (clinical and non-clinical HCW) of our university hospital were able to ask for a consultation at the permanence without an appointment, and not only employees who were in charge of COVID-19 patients.

\section{f. Statistics}

\section{i. Quantitative analysis}

Descriptive statistical analyses were made using Excel ${ }^{\circledR}$ and an $R \circledast$ software package provided by the $R$ Foundation for Statistical Computing. Qualitative variables were expressed as frequencies and percentages. Quantitative variables were expressed as means with minimum and maximum values. Missing data were taken into account for these descriptive analyses and indicated in the corresponding tables (see "n").

\section{i. Qualitative analysis}

We also performed a qualitative analysis of the47 written interview notes of HCW that consented to participate in the study: we systematically analyzed the interviews and looked for frequent themes of 
difficulties expressed by workers. Using content analysis methodology, a coder reviewed all interviews scripts for recurrent themeswhich they then categorized and sub-categorized, while comparing emerging categories to each other to determine their substance and significance [30]. A recurrent theme was defined as a theme occurring more than two times in the interviews of two different HCWs.

\section{Results}

We provided 52 consultations from the $16^{\text {th }}$ march to the $12^{\text {th }}$ of June 2020 and $25 \mathrm{HCW}$ consulted. Of the 25 employees, only 18 gave informed consent,which allowed us to retrospectively analyze their personal and clinical data for the study.

\section{a. CovidPsy consultation activity}

\section{i. Quantitative analysis of clinical data}

The average number of consultations was $(\mu=2.68,1-11)$ and their average duration was 89 minutes. We found $72.22 \%(n=13)$ of HCW consulting at the permanence were frontline health care workers, meaning those who interacted directly with COVID-19-positive, or potentially positive, patients, $77.78 \%$ were women $(n=14), 83.33 \%$ married or living in common law $(n=15), 38.89 \%$ of HCW had a psychiatric history $(n=7)$ and $83.33 \%$ reported entourage support $(n=15)$ which is considered as a protective factor (Table 1$)$.

Average PDI scores were very high $(\mu=28.14,1-51)$, with $11(78.57 \%)$ having PDI scores $\geq 15$ suggesting an important risk of future PTSD, similar to PDEQ scores ( $\mu=21.85,9-39)$, with11(78.57\%) having scores $\geq 15$ (Table 2). In addition, we found high HADS-anxiety scores $(\mu=13.25,6-20)$, with $11(68.75 \%) \geq 11$, suggesting pathological and severe anxiety. The mean HADS-depression scores was less high $(\mu=8.31,1-16)$, with $5(31.25 \%)$ having higher than 11 . Burnout symptoms were very frequent: $4(23.53 \%)$ with severe scores and $6(35.29 \%)$ with moderate scores of Emotional Exhaustion $(\mu(E E)=26.35$, $7-46), 3(17.65 \%)$ with moderate and $4(23.53 \%)$ with severe depersonalization levels, $(\mu(P D)=6.58,1-22)$, $2(11.76 \%)$ with low and $6(35.29 \%)$ with moderate personal achievement levels $(\mu(P A)=31.70,6-45)$, and 7 (38.88\%) with a high level of burnout syndrome according to the MBI-HSS (Table 2, figure 2) . On the basis of our semi-structured interviews, we concluded that 7 (38.89\%) had an ASD or a PTSD diagnosis, and 4 (22.22\%) had an adjustment disorder according to DSM-5 criteria. Furthermore, 1 (5.55\%) had a Major Depressive Episode, and 1(5.55\%) had no psychiatric diagnosis, with some HCW having combined diagnosis (Figure 2). Only $7(38.89 \%)$ HCW had to stop working for a period of time and $5(27.77 \%)$ were referred to a psychiatrist-psychotherapistor a psychologistpsychotherapist follow-up, and $13(72.22 \%)$ did not need to be referred because they were sufficiently clinically improvedafter the end of the CovidPsy consultation. 11(61.11\%) had received an anxiolytic prescription, 7(38.89\%) an antidepressant prescription, and 2 (11.11\%), no prescription of psychotropic medication (Table 1).

ii. Qualitative analysis of clinical data: Difficulties and stress factors during the first wave of the COVID-19 pandemic 
A first category of theme that we called"traumatic stressors" was found and correspond to exposure to multiple deaths, fear of contamination, and feeling insecure. Hospital workers mostly expressed difficulties in coping with uncertainty related to COVID-19, and especially coping with contradictory information about how contamination occurred, and the lack of knowledge about the infection itself. In general, HCW suffered from having to adapt many changes, which createdfeelings of insecurity. $38.89 \%$ were afraid of being contaminated $(n=7)$ and subsequently infecting family members or their social circle(Table 3).

Another category of theme that we called "work related stress" appeared to align with work stressors corresponding to work overload $(33.33 \%, n=6)$, lack of recognition $(27.78 \%, n=5)$, and feelings ofabandonment by their hierarchical superiors $(50 \%, n=9)$, as in this context it was difficult and they expected to be more supported. HCW who received consultation were able toexpress their feelings of shame and their fear of being negatively perceived by their hierarchy or colleagues. This second category of theme that we can call "work related stress" can primarily explain the observed burnout syndrome(Table 3).

\section{Discussion}

The difficulties that we encountered for the implementation of the CovidPsy consultation was in link with the emergency context and having to take very quick decisions to start the consultation. Training sessions were the occasion to have common clinical references for the activity.

HCW who came to the psychiatric consultation constituted a small percentage of overallHCW (25 on $13^{\prime} 557 \mathrm{HCW}$ ). The psychiatric consultation was only one of several strategies implemented during the first wave in terms of the psychological support. Therefore, the first hypothesis to explain the low number of consultations is that HCW felt helped by other implemented strategies (e.g. psychological support provided close to care units, hypnosis sessions, and hotlines). The presence of psychologists within the COVID care units, who could be solicited for speaking in one-to-one settings, or for group interventions,likely played an extremely important role. These interventions were notably different from those coming fromCovidPsy consultation. One could also hypothesize that shame and fear of judgement, due to the stigma of psychiatry, could explain this small number of consultations. Workers expressed that they were reluctant to benefit from a psychiatric consultation within the hospital during working hours as they considered that when they were present, their concentration should be on providing care, suggesting that the hospital setting did not facilitate the use of the permanence. Finally, we can argue that in the heart of the first wave, the vast majority of HCW did not feel the need to ask for help because they were motivated by their goal and their pride to accomplish their mission. During the post-crisis phases and successive COVID-19 waves, one can imagine that psychological support could still have been useful and perhaps more needed with time.The first reason may be a delay in psychic distress from the event, by virtue of an afterthought effect. The second reason relates to possible exhaustion over time theseadditional COVID-19 waves and the absence of any possibility to recover between them. Therefore, we will have to be vigilant about potential long term psychological effects, particularly if we consider the 
prospective disillusionment and reconstruction phases of human services workers following a disaster [31].

In all likelihood, this type of consultation would have been useful following the first wave of COVID-19, however we could not extend it due the resumption of the usual clinical activities of psychiatrists and clinical specialist nurses in psychiatry of the psychiatric consultation.

The high majority of the HCW who came to us, worked in COVID-19 units and were either physicians or nurses, confirming what others have found regarding risk factors of stress related to working in COVID units during this crisis[4] [5].

The burnout and traumatic stress related disorders (ASD or PTSD)suchanxiety and depressive symptoms.Most individuals suffered from working conditions relatedto their own safety, even if they themselves were not considered at risk to develop a severe form of COVID-19. For certain HCW, they did not feel enough supported by their colleagues, and/or hierarchy. In previous studies,the high prevalence of PTSD has been confirmed in several studies certain variables were found to be of particular relevance as risk factors as well as resilience factors, including exposure level, working role, years of work experience, social and work support, job organization, quarantine, age, gender, marital status, and coping styles[6][32].

In our study, participants presented burnout symptoms in line with the lack of recognition and work overload: $35.29 \%$ presented a moderate and $23.53 \%$ a severe emotional exhaustion level, $17.65 \%$ a moderate and $23.53 \%$ a severe depersonalization level, $11.76 \%$ a low and $35.29 \%$ a moderate personal achievement (Figure 1). These results confirm those of an Italian study that showed five weeks after the beginning of the outbreak that almost 33\% presented high scores of Emotional Exhaustion and almost $25 \%$ reported a high levels of Depersonalization [33], witha meta-analysis that identified a burnout prevalence of $37.4 \%$ [5]. Most of them (72.22\%) were sufficiently clinically improvedafter the end of the CovidPsy consultation and we could stop the CovidPsy consultation without referral to a psychiatrist or psychologist.

To the best of our knowledge, there is no previous qualitative study concerning physical psychiatric consultations with HCW during the first wave of COVID-19, most interventions consisting on hotlines [34] [35], and not face-to-face consultations which offer more in-depth care. We show that the qualitative analysis identified subjective information about the difficulties that caused stress symptoms and will be helpful in elaborating preventive and therapeutic strategies. Although we did not obtain follow-up data, we noted only a few participants needed to be referred for psychiatric or psychological follow-ups at the end of the CovidPsy care,which was sufficient for a majority of the HCW who consulted. This suggests that the efficiency of early detection and care of HCW with psychological suffering to reduce long term health and work consequences would need to be confirmed in a prospective study design. The workrelated stress linked with work overload, lack of recognition, and feelings of abandonment by the hierarchy,suggest certain management principles at hospital such as reinforcement of staff during crisis, supporting the efforts of HCW, and accompanying them, are necessary. 
This description of a consultation activity and the psychiatric screening during the first wave cannot be generalized because of its small sample size. Furthermore, qualitative data was collected by only one coder, instead of several persons. In addition, we analyzed interviews that were not exact verbatim records of the consultations, having been transcribed and interpreted by the psychiatrist in a clinical context. Finally, we did not explore protective factors that could have been helpful for HCW during this unprecedented crisis, except in regards to entourage support, and we do not have long-term follow-ups to assess the evolution of the intervention.

\section{Conclusion}

This psychiatric consultation for HCWexperience provides confirmationof the psychiatric consequences during the first wave of COVID-19 and the type of responsesto prevent and early treat potential psychiatric complications. ASD, PTSD, burnout and anxiety symptoms were the most frequent psychiatric outcomes observed. Long-term and psychiatric consequences on mental health are expected in HCW that worked during the first wave ofCOVID-19.

\section{Abbreviations}

Acute stress disorder (ASD)

Cognitive-Behavioral Therapies (CBT)

COronaVIrus Disease-19 (COVID-19)

Depersonalization (DP)

Diagnostic and Statistical Manual of Mental Disorders (DSM-5)

Emotional Exhaustion (EE)

Eyes Movement Desensitization Reprocessing (EMDR)

Healthcare workers $(\mathrm{HCW})$

Hospital Anxiety and Depression Scale (HADS)

Maslach Burnout Inventory-Human Services Survey (MBI-HSS)

Middle East Respiratory Syndrome (MERS)

Peritraumatic Dissociative Experiences Questionnaire (PDEQ)

Peritraumatic distress inventory (PDI)

Personal Achievement (PA) 
Post-Traumatic Stress Disorder (PTSD)

Posttraumatic Stress disorder Checklist for DSM-5 (PCL-5)

Severe Acute Respiratory Syndrome (SARS)

\section{Declarations}

\section{Ethics approval and consent to participate}

To retrospectively analyze personal data, we asked all HCW who consulted at the CovidPsy consultation between the $19^{\text {th }}$ March 2020 and the $12^{\text {th }}$ June 2020 for theirinformed consent in accordance with the decision of august the $20^{\text {th }} 2020$ of the Cantonal Commission for the Ethics of Research on Human Beings of Geneva, an official commission of the State of Geneva ( $\left.n^{\circ} 2020-02036\right)$. The need for ethics approval was waived by this Commission. We obtained a written consent from all the participants but no administrative permissions were required to access and use the dataset/medical records.

\section{Consent for publication}

We get a written consent for publication from all the participants.

\section{Availability of data and materials}

The datasets generated and analysed during the current study are not publicly available due to the fact that we can't share identifying and confidential patient data.

\section{Competing interests}

The authors declare that they have no competing interests.

\section{Funding}

There is no funding for this study.

\section{Author's contributions}

DA, VM and LB asked for ethics approval. AG and CC informed participants and obtained informed consents.DA, CC and LB conducted psychiatric interviews. DA, CC, LB, GL, BC, AG, VM provided psychotherapeutic interventions. LB and GB analyzed and interpreted the HCW socio-demographic and clinical data and were major contributors in writing the manuscript. All the authors read and approved the final manuscript.

\section{Aknowledgements}

We aknowledge Jeffrey Hubbard for his proofreading of the English language. 


\section{References}

1. John Hopkins University of Medicine. Coronavirus Ressource Center. https://coronavirus.jhu.edu/map.html. Accessed 17 May 2021

2. [Office fédéral de la Santé Publique]. [Cas confirmés en laboratoire]. https://www.covid19.admin.ch/fr/epidemiologic/case. Accessed 17 May 2021

3. World Health Organization. Interim Briefing Note- Taking into account the psychosocial and mental health aspects of the Covid-19 epidemic. 2020.https://interagencystandingcommittee.org/iascreferencegroup-mental-health-and-psychosocial-support-emergency-settings/interim-briefing . Accessed 17 March2020.

4. Pappa S, Ntella V, Giannakas T, Giannakoulis VG, Papoutsi E, Katsaounou P. Prevalence of depression, anxiety, and insomnia among healthcare workers during the COVID-19 pandemic: A systematic review and meta-analysis. Brain Behav Immun. 2020; Aug;88:901-907.

5. Batra K, Singh TP, Sharma M, Batra R, Schvaneveldt N. Investigating the Psychological Impact of COVID-19 among Healthcare Workers: A Meta-Analysis. Int J Environ Res Public Health. 2020;Dec 5;17(23):9096.

6. Carmassi C, Foghi C, Dell'Oste V, Cordone A, Bertelloni CA, Bui E, Dell'Osso L. PTSD symptoms in healthcare workers facing the three coronavirus outbreaks: What can we expect after the COVID-19 pandemic. Psychiatry Res. 2020 Oct; 292:113312.

7. Maves RC, Downar J, Dichter JR, Hick JL, Devereaux A, Geiling JA, Kissoon N, Hupert N, Niven AS, King MA, Rubinson LL, Hanfling D, Hodge JG Jr, Marshall MF, Fischkoff K, Evans LE, Tonelli MR, Wax RS, Seda G, Parrish JS, Truog RD, Sprung CL, Christian MD; ACCP Task Force for Mass Critical Care. Triage of Scarce Critical Care Resources in COVID-19 An Implementation Guide for Regional Allocation: An Expert Panel Report of the Task Force for Mass Critical Care and the American College of Chest Physicians. Chest. 2020; Jul;158(1):212-225.

8. Maslach C, Schaufeli WB, Leiter MP. Job burnout. Annu Rev Psychol. 2001;52:397-422.

9. Rotenstein LS, Torre M, Ramos MA, Rosales RC, Guille C, Sen S, Mata DA. Prevalence of Burnout Among Physicians: A Systematic Review. JAMA. 2018; Sep 18;320(11):1131-1150.

10. West CP, Dyrbye LN, Shanafelt TD. Physician burnout: contributors, consequences and solutions. J Intern Med. 2018 Jun;283(6):516-529. doi: 10.1111/joim.12752. Epub 2018 Mar 24. PMID: 29505159.

11. American Psychiatric Association, Diagnostic and statistical manual of mental disorders. Fifth ed. American Psychiatric Association Publishing, Washington ; 2013

12. Brewin $C R$, Andrews $B$, Valentine JD. Meta-analysis of risk factors for posttraumatic stress disorder in trauma-exposed adults. J Consult Clin Psychol e2000 Oct;68(5):748-66.

13. Bai Y., Lin C.C., Lin C.Y., Chen J.Y., Chue C.M., Chou P. Survey of stress reactions among health care workers involved with the SARS outbreak. Serv. 2004;55(9):1055-1057

14. Watson P. PTSD as a Public Mental Health Priority. Curr Psychiatry Rep. 2019; Jun 26;21(7):61. 
15. Qi W, Gevonden M, Shalev A. Prevention of Post-Traumatic Stress Disorder After Trauma: Current Evidence and Future Directions. Curr Psychiatry Rep. 2016; Feb;18(2):20.

16. Roberts NP, Kitchiner NJ, Kenardy J, Lewis CE, Bisson JI. Early psychological intervention following recent trauma: A systematic review and meta-analysis. Eur J Psychotraumatol. 2019; Dec 6;10(1):1695486.

17. Brooks S, Amlôt R, Rubin GJ, Greenberg N. Psychological resilience and post-traumatic growth in disaster-exposed organisations: overview of the literature. BMJ Mil Health. 2020; Feb;166(1):52-56.

18. World Health Organization.. Support based on psychological first aid principles in people recently exposed to a traumatic event. 2012. In: WHO mhGAP Action Programme who.int/mental_health/mhgap/evidence/other_disorders/q6/en/ .Accessed May 13,2020.

19. Norwood AE, Ursano RJ, Fullerton CS. Disaster psychiatry: principles and practice. Psychiatr Q. 2000; Fall;71(3):207-26.

20. Nussbaum A, The Pocket Guide to the DSM-5 ${ }^{\mathrm{TM}}$ Diagnostic Exam, American Psychiatric Publishing, Inc, 2013.

21. Burnout Inventory Manual. 2nd edition. Palo Alto, CA: Consulting Psychologists Press, 1986.

22. Faye-Dumanget $C$, Carré J, Le Borgne $M$, Boudoukha PAH. French validation of the Maslach Burnout Inventory-Student Survey (MBI-SS). J Eval Clin Pract. 2017 Dec;23(6):1247.

23. Zigmond AS, \& Snaith R P. The hospital anxiety and depression scale. Acta psychiatrica Scandinavica.1983 : https://doi.org/10.1111/j.1600-0447.1983.tb09716.x

24. Brunet A, Weiss DS, Metzler TJ, Best SR, Neylan TC, Rogers C, Fagan J, Marmar CR. The Peritraumatic Distress Inventory: A proposed Measure of PTSD criterion A2. American Journal of Psychiatry.2001;158, 1480-1485.

25. Jehel, L., Brunet, A., Paterniti, S., Guelfi JD. Validation of the French version of the Peritraumatic Distress Inventory. Canadian Journal of Psychiatry. 2005;50, 67-71.

26. Marmar CR., Weiss DS, \& Metzler TJ. The Peritraumatic Dissociative Experiences Questionnaire. In : Wilson JP, Marmar CR, editors. Assessing psychological trauma and posttraumatic stress disorder. New York : The Guilford Press; 1997.pp. 412-428.

27. Birmes P, Brunet A, Benoit M, Defer S, Hatton L, Sztulman H, Schmitt L. Validation of the Peritraumatic Dissociative Experiences Questionnaire self-report version in two samples of Frenchspeaking individuals exposed to trauma. European Psychiatry. 2005 Mar;20(2):145-51.

28. Ashbaugh, AR., Houle-Johnson S, Herbert C, El-Hage W, \& Brunet A. Psychometric Validation of the English and French Versions of the Posttraumatic Stress Disorder Checklist for DSM-5 (PCL-5). 2016; PloS one, 11(10), e0161645.

29. Weathers FW, Litz BT, Keane TM, Palmieri PA, Marx BP, \& Schnurr, PP 2013. The PTSD Checklist for DSM-5 (PCL-5).Retrieved from ptsd.va.gov. Accessed 19 May 2021.

30. Hsieh H-F, Shannon, SE Three Approaches to qualitative content analysis. Qual Health Res. 2005; Nov;15(9):1277-88. 
31. Substances abuses and Mental Health Services Administration. Phases of disaster. https://www.samhsa.gov/dtac/recovering-disasters/phases-disaster. Accessed 17 May 2021

32. Di Tella M, Romeo A, Benfante A, Castelli L. Mental health of healthcare workers during the COVID-19 pandemic in Italy. J Eval Clin Pract. 2020 Dec;26(6):1583-1587.

33. Barello S, Palamenghi L, Graffigna G. Burnout and somatic symptoms among frontline healthcare professionals at the peak of the Italian COVID-19 pandemic. Psychiatry Res. 2020; Aug;290:113129

34. Geoffroy PA, Le Goanvic V, Sabbagh O, Richoux C, Weinstein A, Dufayet G, Lejoyeux M. Psychological Support System for Hospital Workers During the Covid-19 Outbreak: Rapid Design and Implementation of the Covid-Psy Hotline. Front Psychiatry. 2020 May 28;11:511.

35. Rolling J, Mengin AC, Palacio C, Mastelli D, Fath M, Gras A, Von Hunolstein JJ, Schröder CM, Vidailhet P. COVID-19: Mental Health Prevention and Care for Healthcare Professionals. Front Psychiatry. 2021 Mar 23;12:566740.

\section{Tables}

Table 1: Socio-demographic characteristics, clinical data and permanence activity 


\begin{tabular}{|c|c|}
\hline & $\begin{array}{l}\text { Total } \\
(\mathrm{N}=18) ; \mathrm{N}(\%)\end{array}$ \\
\hline Age (mean, min-max) & $40,67(25-58)$ \\
\hline \multicolumn{2}{|l|}{ Gender } \\
\hline Female & $14(77.78)$ \\
\hline Male & $4(22.22)$ \\
\hline \multicolumn{2}{|l|}{ Profession } \\
\hline Physician & $2(11.11)$ \\
\hline Nurse & $9(50)$ \\
\hline Medical student & $2(11.11)$ \\
\hline Other healthcare worker & $4(22.22)$ \\
\hline Administrative hospital staff & 1(5.55) \\
\hline \multicolumn{2}{|l|}{ Marital status } \\
\hline Single & $3(16.67)$ \\
\hline Married or living as a cople & 15(83.33) \\
\hline \multicolumn{2}{|l|}{ Entourage support } \\
\hline Yes & 15(83.33) \\
\hline No & $3(16.67)$ \\
\hline \multicolumn{2}{|l|}{ Working in COVID yard } \\
\hline Yes & $13(72.22)$ \\
\hline No & $5(27.78)$ \\
\hline \multicolumn{2}{|l|}{ Medical hisory } \\
\hline Yes & $8(44.44)$ \\
\hline No & $7(55.56)$ \\
\hline \multicolumn{2}{|l|}{ Psychiatric history } \\
\hline Yes & $7(38.89)$ \\
\hline No & $11(61.11)$ \\
\hline - $\quad$ No information & \\
\hline Current psychotrop medication & \\
\hline
\end{tabular}




\begin{tabular}{|c|c|c|}
\hline - & Yes & $2(11.11)$ \\
\hline- & No & $16(88.89)$ \\
\hline \multicolumn{3}{|c|}{ Trauma history } \\
\hline- & Yes & $9(50)$ \\
\hline- & No & $9(50)$ \\
\hline \multicolumn{3}{|c|}{ SARS-CoV-2 contamination } \\
\hline- & Yes & $4(22.22)$ \\
\hline- & No & $14(77.78)$ \\
\hline \multicolumn{3}{|c|}{ Medical risk factors for severe COVID-19 form* } \\
\hline- & Yes & $2(11.11)$ \\
\hline- & No & 16(88.89) \\
\hline & Number of consultations/number of consultations by HCW & $52 / \mu=2.68,1-11$ \\
\hline & ion of consultation & $\begin{array}{l}\mu=89 \min (\min \text { and } \max \text { value } \\
\text { unknown) }\end{array}$ \\
\hline \multicolumn{3}{|c|}{ Referral to a psychiatrist or a psychologist follow-up } \\
\hline - & Yes & $5(27.78)$ \\
\hline- & No & $13(72.22)$ \\
\hline \multicolumn{3}{|c|}{ Sick leave } \\
\hline- & Yes & $7(38.89)$ \\
\hline- & No & $11(61.11)$ \\
\hline \multicolumn{3}{|c|}{$\begin{array}{l}\text { Prescription of a psychotropic medication in the contexte of } \\
\text { CovidPsy consultation }\end{array}$} \\
\hline- & Anxiolytic medication & $11(61.11)$ \\
\hline - & Antidepressant medication & $2(11.11)$ \\
\hline- & No psychotrop prescription & $7(38.89)$ \\
\hline
\end{tabular}

*diabetes, obesity, hypertension, history of heart failure, ischaemic heart disease, solid organ tumours, chronic obstructive pulmonary disease (COPD), chronic respiratory disease, chronic.

Table 2: Results of PDEQ, PDI, PCL-5, HADS and MBI-SS scales. 


\begin{tabular}{|c|c|}
\hline \multicolumn{2}{|l|}{ PDEQ $(n=14)$} \\
\hline PDEQ score (mean, min-max) & $21.85(9-39)$ \\
\hline Positive PDEQ $(\mathrm{N}, \%))$ & 11(78.57) \\
\hline \multicolumn{2}{|l|}{ PDI (n=14) } \\
\hline PDI score (mean, min-max) & $28.14(1-51)$ \\
\hline Positive PDI (N,\%) & 11(78.57) \\
\hline PCL-5 (n=4) & \\
\hline Positive PCL-5 (N, \%) & $4(100)$ \\
\hline PCL-5 (mean, min-max) & $30(33-47)$ \\
\hline \multicolumn{2}{|l|}{ HADS-A $(n=16)$} \\
\hline Positive HADS-A (N, \%) & $11(68.75))$ \\
\hline HADS-A (mean, min-max) & $13.25(6-20)$ \\
\hline \multicolumn{2}{|l|}{ HADS-D $(n=16)$} \\
\hline Positive HADS-D (N, \%) & $5(31.25)$ \\
\hline HADS-D (mean, min-max) & $8.31(1-16)$ \\
\hline \multicolumn{2}{|l|}{ MBI-HSS (n=17) } \\
\hline MBI-EE (N, \%) & \\
\hline o Low level (sub-score $\leq 21)(\mathrm{N}, \%)$ & $7(41.8)$ \\
\hline o Moderate $(21<$ sub-score $\leq 32)$ & $6(35.29)$ \\
\hline o High level (sub-score>32 & $4(23.53)$ \\
\hline - $\quad \operatorname{MBI-DP}(\mathrm{N}, \%)$ & \\
\hline o Low level (sub-score $\leq 6$ ) & $10(58.82)$ \\
\hline o Moderate $(21<$ sub-score $\leq 12$ & $3(17.65)$ \\
\hline o High level (>12) & $4(23.53)$ \\
\hline - $\quad$ MBI-PA (N, \%) & \\
\hline o Low level (sub-score $\leq 22$ ) & $2(11.76)$ \\
\hline o Moderate $(22<$ sub-score $\leq 32)$ & $6(35.29)$ \\
\hline o High level (sub-score >32) & $9(52.94)$ \\
\hline
\end{tabular}


Table 3: Qualitative analysis of the 47 written interview notes with HCW.

\begin{tabular}{|c|c|c|}
\hline \multicolumn{2}{|r|}{ Percieved difficulties at work in link with COVID-19 outbreak ( $N=18)$} & $N(\%)$ \\
\hline \multicolumn{3}{|c|}{ Traumatic stressors } \\
\hline- & Fear of contamination at work & $7(38.89)$ \\
\hline- & Feeling insecure & 6(33.33) \\
\hline- & Multiple deaths of patients & $5(27.78)$ \\
\hline \multicolumn{3}{|c|}{ Work related stress } \\
\hline- & Work overload & $6(33.33)$ \\
\hline- & Lack of recognition & $5(27.78)$ \\
\hline - & Feeling abandoned by hierarchy & $9(50)$ \\
\hline
\end{tabular}

\section{Figures}

\begin{tabular}{|l} 
Emotional exhaustion Depersonalization (DP) Pchievement (PA) Penal A \\
ModerateEE
\end{tabular}

\section{Figure 1}

Three dimensions of MBI-HSS distribution. 


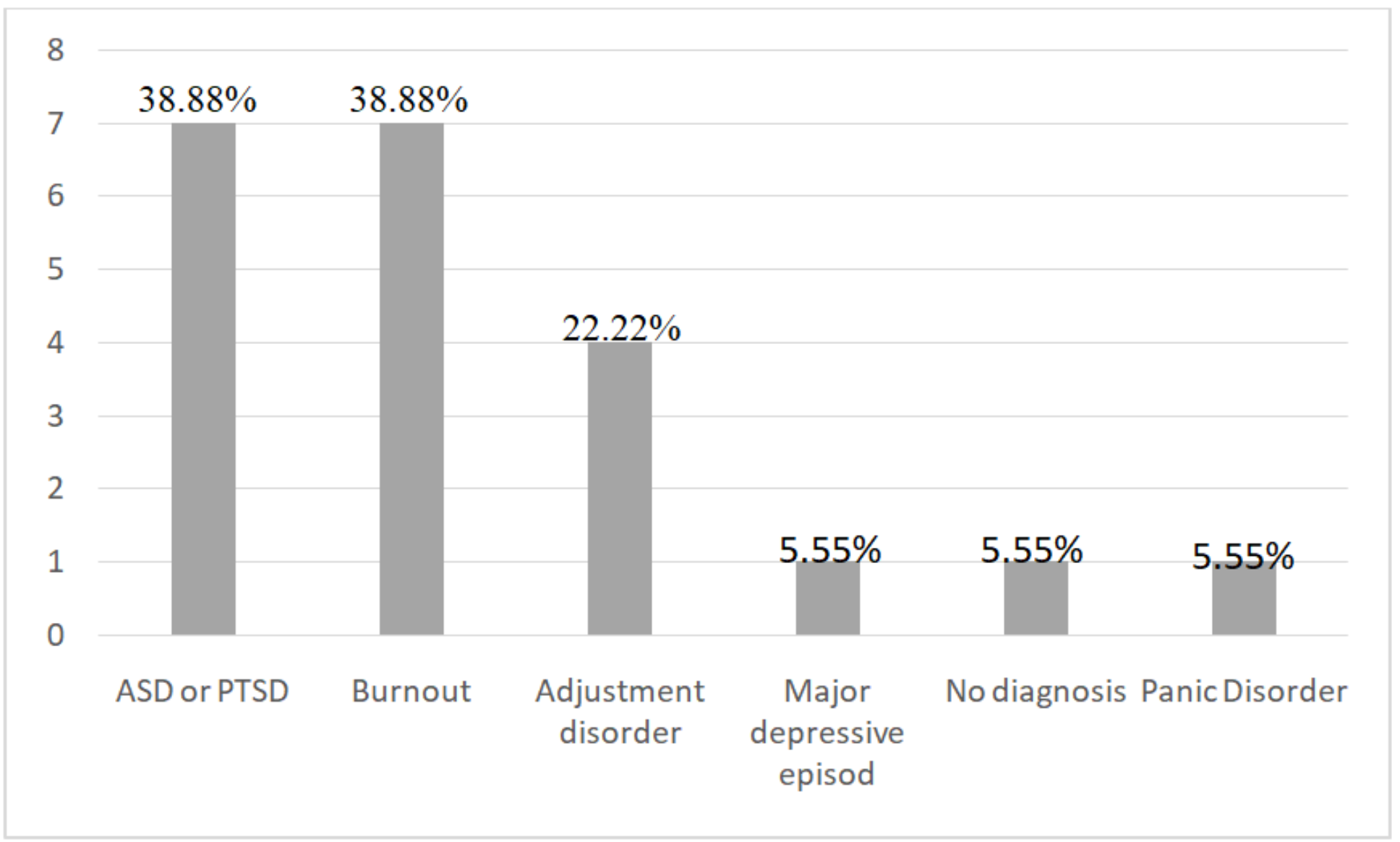

Figure 2

Psychiatric diagnosis retained according to DSM-5 criteria diagnosis and MBI-HSS. 